\title{
Integration of Wearable Devices in Healthcare
}

\author{
Tanaya Jain ${ }^{1}$, Ranjit Ambad ${ }^{2}$, Prajakta Warjukar ${ }^{3}$ and Nandkishor Bankar ${ }^{5}$ \\ ${ }^{1}$ First Year MBBS Student Datta Meghe Medical College, Nagpur, India \\ ${ }^{2}$ Department of Biochemistry Datta Meghe Medical College, Nagpur, India \\ ${ }^{4}$ Department of Biochemistry Datta Meghe Medical College, Nagpur, India \\ ${ }^{5}$ Dept. of Microbiology Jawaharlal Nehru Medical College, Datta Meghe Institute of \\ Medical Sciences Sawangi (Meghe) Wardha, India
}

\section{ABSTRACT}

Smart devices play vital role due to their responsiveness to stimuli like heat, temperature, $\mathrm{pH}$, stress in our lives. These devices have changed our lives as compared to our ancestors. Wearable devices need energy to function which can be provided by batteries but recharging them timely is not feasible so energy to run these devices is harnessed from the mechanical energy of the body. Wearable device should have less Young's modulus and elastic limit should be high. These devices can be integrated into items of daily use like bracelets, armbands, wristwatches, rings, vests, shoes and gloves. Wearable devices provide numerical or graphical data via sensors.

\section{KEY WORDS: WEARABLE DEVICES, ARTIFICIAL INTELLIGENCE.}

\section{INTRODUCTION}

There is surge in chronic degenerative diseases in population and this has boosted the demands of wearable medical devices. Their applications are monitoring, diagnosis, drug administration, surgery. These medical smart devices have transducers which are to be placed in contact of skin, microcontrollers, transceivers. The materials used should be biocompatible, durable, flexible, washable. These systems are easy to carry and also provide GPS location with real time records. They reduce hospital visits.

Parameters that can be monitored are: Heart rate - heart rate is frequency of cardiac cycle measured in bpm. It can detect bradycardia or tachycardia.

Respiratory rate - for detection of respiratory rate devices are mounted on chest, abdomen. Abnormal respiratory

Biosc Biotech Res Comm P-ISSN: 0974-6455 E-ISSN: 2321-4007
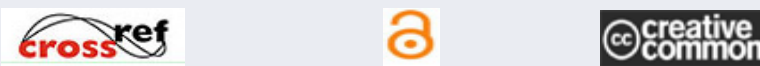

Identifiers and Pagination Article Information

Year: 2021 Vol: 14 No (7) Special Issue

Pages: 131-132

Received: $14^{\text {th }}$ April 2021

This is an open access article under Creative

Commons License Attribn 4.0 Intl (CC-BY).

DOI: $h t t p: / / d x$.doi.org/10.21786/bbrc/14.7.32 rate can suggest asthma, dyspnoea, bradypnoea, tachypnoea, cheye-stokes syndrome, apnoea.

Blood pressure- smartwatches, smartglasses with optical sensors are used to measure the blood pressure and circuit board is attached to finger and they provide real time monitoring of pressure changes.

Parkinson tremors- the wearable devices like gloves reduce the amplitude of tremors and can also suppress them.

Heart attacks- smart textiles are effective method to monitor heart attacks.

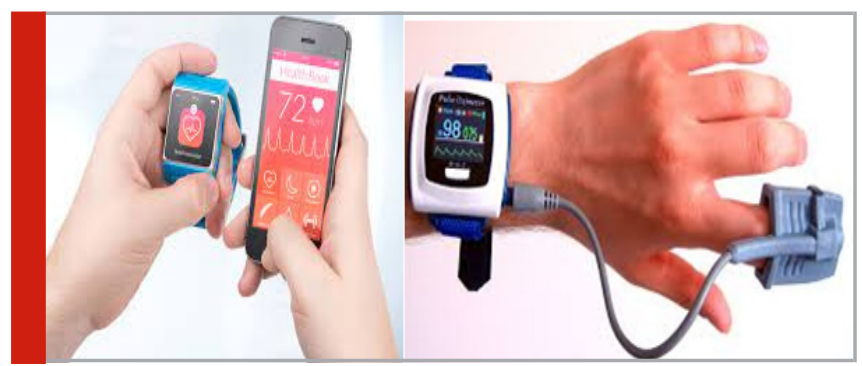




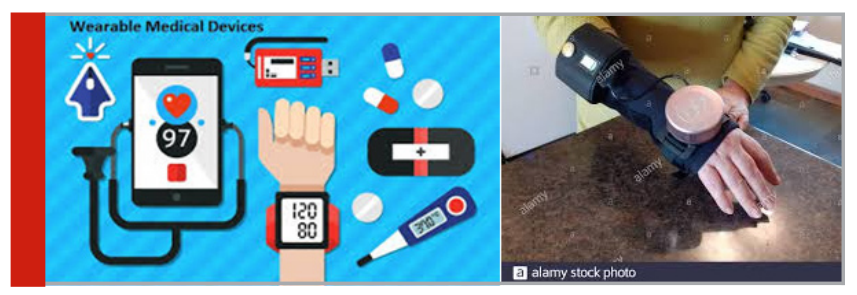

\section{CONCLUSION}

Advancement in healthcare system can be seen with due integration of IoT which has increased the quality of disease management system, patient care, patient experience and precision and also effective in cost reduction. Smart healthcare wearables are combination of IoT, 5G network, cloud computing, artificial intelligence, biotechnology. Wearable devices give accurate, efficient, timely data for therapeutic use \&t disease management.

\section{REFERENCES}

Smart materials for smart healthcare- moving from sensors and actuators to self-sustained nonenergy nan.o systems,

IoT Based Wearable Device to Monitor the Signs of Quarantined remote patients of COVID-19., Nizar Al
Bassam, Hussain Shaik Asif, Ammar Al Qaraghuli, Jibreal Khan, E.P. Sumesh, Vidhya Lavanya, Informatics in Medicine Unlocked

Flexible ferroelectric wearable devices for medical applications.

A review of wearable internet of things device for heathcare.

Smart wearable body sensors for patient self assessment and monitoring.

Computer science department, BINUS graduate program, master of computer science, Bina Nusantara, University, Jakarta, Indonesia-11480.

Faezeh Arab Hassani, Qiongfeng Shi, Feng Wen, Tianyiyi He, Ahmed Haroun, Yanqin Yang, Yuqin Feng, Chengkuo Lee,

Geoff Appelboom, Elvis Camocho, Mickey E. Abraham, Samuel S. Bruce, Emmanuel LP Dumont, Brad E. Zacharia, Randy D'Amico, Justin Slomian, Jean Yve Reginster, Olivier Bruyere, E Sander Connolly Jr.

Nico Surantha, Prabadinata Atmaja, David, Maulana Wicaksono.

Smart Materials in Medicine

Zois Michail Tsikriteas, James I. Roscow, Chris R. Bowmen, Hamideh Khanbareh 